\title{
Thermal Stability: The Next Frontier for Nanocrystalline Materials
}

\author{
SUVEEN N. MATHAUDHU ${ }^{1,2,4,5}$ and BRAD L. BOYCE ${ }^{3,6}$ \\ 1.-Department of Mechanical Engineering, University of California-Riverside, Riverside, CA, \\ USA. 2.-Energy and Environment Directorate, Pacific Northwest National Laboratory, Richland, \\ WA, USA. 3.-Sandia National Laboratories, Albuquerque, NM, USA. 4.-e-mail: smath- \\ audhu@engr.ucr.edu. 5.—e-mail: suveen.mathaudhu@pnnl.gov. 6.—e-mail: blboyce@sandia.gov
}

For the past quarter decade, the science and technology of nanocrystalline materials (materials with grain sizes less than $100 \mathrm{~nm}$ ) has been an extremely rich and diverse field of study. ${ }^{1,2}$ Generally, it has been observed that tremendous improvements in physical and mechanical properties, including order-of-magnitude increases in yield strength, are possible. ${ }^{2}$ As predicted by the HallPetch equation, ${ }^{3,4}$ a reduction in grain size should be accompanied by an increase in strength. But, despite the promise of nanocrystalline materials for a host of structural and functional applications, their use has been severely limited by their lack of microstructural stability at elevated temperatures ${ }^{5}$ or under mechanical loads. ${ }^{6,7}$ In the case of pure metals, this coarsening often occurs even at ambient temperatures. ${ }^{5}$ Ironically, the same features that often result in the enhancement of properties in nanocrystalline materials, namely the high volume fraction of high-energy grain boundaries, are responsible for the observed grain growth or phase transformation. ${ }^{8}$ From this observation, it follows that control of the grain boundaries and interfacial regions provides the key to retention of the nanocrystalline grain size and phase stability at the high temperatures observed in both the manufacturing process and in the service life of the component.

An illustration of this principle can be demonstrated via the equation for the velocity of a grain boundary:

$$
v=M P
$$

where $M$ is the boundary mobility and $P$ is a driving force for boundary motion. The basis of this concept can be traced back to origins in the work of

Suveen N. Mathaudhu and Brad L. Boyce are the guest editors for the Mechanical Behavior of Materials Committee of the TMS Structural Materials Division; and coordinators of the topic Stability of Nanomaterials in this issue.
Turnbull. ${ }^{9}$ In a simplistic sense, the mobility represents the kinetics and the driving force represents the thermodynamics. There are many variations of this basic premise, with the most common including an Arrhenius activation of the mobility term and driving force represented by excess interfacial energy due to curvature of the boundaries ${ }^{10}$ :

$$
v=M P=M_{0} \exp \left[\frac{-Q_{\mathrm{m}}}{R T}\right] \frac{C \gamma}{r}
$$

where $Q_{\mathrm{m}}$ is the activation energy, $r$ is the grain radius and $\gamma$ is the interfacial energy of the boundary. $M_{0}$ and $C$ are experimental constants, and $R$ is the gas constant. Reduction of the velocity of the grain boundary can be accomplished via reduction of $M$ or $P$; however, a number of factors should be observed. Firstly, a nanocrystalline grain size will result in a very high pressure term that will drive grain growth. Secondly, the mobility term is temperature-dependent, and elevated temperatures will increase the grain boundary mobility. Lastly, if the interfacial energy can be reduced, theoretically the grain boundary energy should approach zero and limit grain growth independent of temperature or grain size. Historically, the mobility of the grain boundary has been limited via the addition of solutes or precipitates that serve as grain boundary pinners. However, given the sensitivity of the mobility to the thermal activation, this approach becomes less tractable with increasing temperatures. More recent studies have investigated the possibility of lowering the interfacial energy via predictive solute segregations, and, furthermore, these approaches can also theoretically consider systems that are not prone to formation of precipitates or secondary phases at elevated temperatures. ${ }^{11,12}$

In this set of manuscripts, we expand on the nascent work in this area and present the state-ofthe-science of thermal stability from many of the 
leading research groups attacking this complex problem. A variety of novel processing approaches are presented for maintaining the grain size of nanocrystalline materials under elevated temperatures, and are summarized here.

Khaljhedayati and Rupert, in a paper entitled "High-Temperature Stability and Grain Boundary Complexion Formation in a Nanocrystalline $\mathrm{Cu}-\mathrm{Zr}$ Alloy", investigate the thermal stability of a mechanically alloyed and annealed $\mathrm{Cu}-\mathrm{Zr}$ nanocrystalline alloy with an amorphous intergranular region. Stability in this case was enabled via reduction of the grain boundary energy via complexion formation and some precipitation of $\mathrm{ZrC}$ particles. Hornbuckle et al. also investigate a $\mathrm{Cu}$ alloy in "Effect of Ta Solute Concentration on the Microstructural Evolution in Immiscible $\mathrm{Cu}-\mathrm{Ta}$ Alloys", wherein they report experimental observations and molecular dynamics simulations. In prior manuscripts, the authors have reported a reduction in the grain boundary interfacial energy due to the segration of $\mathrm{Ta}^{13,14}$ This study expands on that work and reports that the mechanisms for thermal stability in this system are likely temperature-dependent, with the grain boundary Ta solutes playing a role in the stability at lower temperatures, and segregated Ta clusters providing Zener pinning at higher temperatures.

McCabe et al. report on immiscible $\mathrm{Cu}-\mathrm{Nb}$ and $\mathrm{Zr}-\mathrm{Nb}$ composites in "Recrystallization and Grain Growth in Accumulative Roll-Bonded Metal Composites." The accumulative roll-bonding process produced nanocrystalline laminates rather than nanocrystalline grains. The authors seek to understand the driving force for grain growth and recrystallization created by varying the formation and redistribution of dislocations, grain boundaries and phase boundaries during processing. The authors report that phase segregation of the insoluble layers plays a major role in limiting recrystallization and grain growth within the individual layers through a reduction in phase boundary energy.

Komarasamy et al. also delve into the thermal stability of immiscible alloys in "Friction Stir-Processed Thermally Stable Immiscible Nanostructured Alloys." In this study, they observe that the high shear conditions observed during friction stir processing result in enhanced miscibility and solid solution formation with grain refinement to the nanoscale, and phase segregation into Zener pinning nanoprecipitates. These observations bear similarity to those of Hornbuckle et al. Pozuelo et al. similarly investigate an approach relying on grain boundary pinning in "Thermally Stable Nanostructured Magnesium Nanocomposites Reinforced by Diamantane." Herein, they show that Mg-10Al-1 Diamantane (wt.\%) produced by cryomilling plus spark plasma sintering show increased stability via restriction of both $\mathrm{Al}$ diffusion and grain boundary mobility with the diamantane.
Lastly, Kalidindi et al. provide a thorough review of the thermodynamic considerations in "Nanocrystalline Materials at Equilibrium: A Thermodynamic Review." In this review, they present the thermodynamic stabilization approach with an eye towards both stability against grain growth and stability against phase separation. Various thermodynamic models to determine equilibrium states are surveyed, and particular emphasis is placed on emerging meso-scale Monte Carlo simulations and the benefits they provide over atomistic simulations alone.

These recent works present a concise picture of the state of thermal stability research, and exemplify how quickly the prospects for elevated temperature processing and application have improved. The studies also reveal some consistent themes, and uncover some outstanding forecasts for future studies:

- Firstly, it would appear that plenty of opportunity exists to deconvolute the thermodynamic and kinetic contributions to thermal stability. In most of the systems reported, it is challenging to attribute the observed thermal stability solely to one of the mechanisms, and thus the combinatorial effects deserve more study, both experimentally with in situ characterization tools and with simulations. Ultimately, several theories postulate that it should be possible to achieve a chemically enriched grain boundary with no thermodynamic driving force for boundary motion and hence intrinsic stability with no kinetics for motion. However, such an observation has yet to be reported.

- Secondly, the prospects for enabling thermally stable nanocrystalline materials via controlled processing and segregation of immiscible systems are strong. The reports presented show preliminary evidence that control of these phenomena can tailor the diffusion-controlled phenomena that drive grain growth and thus the thermal stability mechanisms.

- Thirdly, as pointed out by Kalidindi et al., opportunities exist to develop computational simulation tools that expand alloy space to incorporate more complex nanocrystalline systems, such as negative enthalpy of mixing compound formers. Unlike analytic models, which typically treat the grain boundaries with homogeneous single-valued properties, simulation could begin to account for the true structural and chemical heterogeneity of boundary types within a material.

- Lastly, while thermal stability has been improved and property retention has been demonstrated after prolonged times at high homologous temperatures, review of the extant literature reveals a large gap in the understanding of the stability of the nanocrystalline materials under mechanical loading, wherein stress 
or strain may drive the movement of grain boundaries, even at low homologous temperatures.

To close, we hope that the readers find this collection of articles interesting and informative. Most importantly, these articles and the numerous others on this subject matter set the stage for what may be a profound breakthrough in materials science: nanostructured metals that retain their superior properties under extreme thermal and mechanical duress.

The following papers being published under the topic of Stability of Nanomaterials provide excellent details and research on the subject. To download any of the papers, follow the url http://link.springer. com/journal/11837/67/12/page/1 to the table of contents page for the December 2015 issue (vol. 67, no. 12).

- "High-Temperature Stability and Grain Boundary Complexion Formation in a Nanocrystalline $\mathrm{Cu}-\mathrm{Zr}$ Alloy" by Amirhossein Khalajhedayati and Timothy J. Rupert.

- "Effect of Ta Solute Concentration on the Microstructural Evolution in Immiscible $\mathrm{Cu}-\mathrm{Ta}$ Alloys" by B.C. Hornbuckle, T. Rojhirunsakool, M. Rajagopalan, T. Alam, G.P. Purja Pun, R. Banerjee, K.N. Solanki, Y. Mishin, L.J. Kecskes, and K.A. Darling.

- "Recrystallization and Grain Growth in Accumulative Roll-Bonded Metal Composites" by Rodney J. McCabe, John S. Carpenter, Sven Vogel, Nathan A. Mara, and Irene J. Beyerlein.

- "Friction Stir-Processed Thermally Stable Immiscible Nanostructured Alloys" by Mageshwari Komarasamy, Rajiv S. Mishra, Sundeep Mukherjee, and Marcus L. Young.

- "Thermally Stable Nanostructured Magnesium Nanocomposites Reinforced by Diamantane" by Y.W. Chang, M. Pozuelo, and J.M. Yang.
- "Nanocrystalline Materials at Equilibrium: A Thermodynamic Review" by Arvind R. Kalidindi, Tongjai Chookajorn, and Christopher A. Schuh.

\section{ACKNOWLEDGMENTS}

BLB was supported by the U.S. Department of Energy, Office of Basic Energy Sciences. Sandia is a multiprogram laboratory operated by Sandia Corporation, a Lockheed Martin Company, for the United States Department of Energy's National Nuclear Security Administration under contract DE-AC04-94AL85000. SNM was supported in part by the Materials Synthesis and Simulation Across Scales $\left(\mathrm{MS}^{3}\right)$ Initiative, a Laboratory Directed Research and Development Program at Pacific Northwest National Laboratory (PNNL), and in part by the University of California, Riverside.

\section{REFERENCES}

1. H. Gleiter, Prog. Mater. Sci. 33, 223 (1989).

2. M.A. Meyers, A. Mishra, and D.J. Benson, Prog. Mater. Sci. 51, 427 (2006).

3. E.O. Hall, Proc. R. Soc. Lond. B Biol. Sci. 64, 474 (1951).

4. N.J. Petch, J. Iron Steel Inst. 174, 25 (1953).

5. V.Y. Gertsman and R. Birringer, Scripta Mat. 30, 577 (1994).

6. T.J. Rupert, D.S. Gianola, Y. Gan, and K.J. Hemker, Science 326, 1686 (2009).

7. K. Zhang, J.R. Weertman, and J.A. Eastman, Appl. Phys. Lett. 85, 5197 (2004).

8. J.R. Weertman, Science 337, 921 (2012).

9. J.E. Burke and D. Turnbull, Prog. Metal. Phys. 3, 220 (1952).

10. C.E. Krill, H. Ehrhardt, and R. Birrenger, Z. Metallk. 96, 1134 (2005).

11. T. Chookajorn, H.A. Murdoch, and C.A. Schuh, Science 337, 951 (2012).

12. M. Saber, C.C. Koch, and R.O. Scattergood, Mater. Res. Lett. 3,65 (2015).

13. K.A. Darling, A.J. Roberts, Y. Mishin, S.N. Mathaudhu, and L.J. Kecskes, J. Alloys Comp. 537, 142 (2013).

14. T. Frolov, K.A. Darling, L.J. Kecskes, and Y. Mishin, Acta Mater. 60, 2158 (2012). 